\title{
Lilian Thuram, Mes étoiles noires. De Lucy à Barack Obama
}

\section{Carminella Biondi}

\section{Q OpenEdition}

1 Journals

\section{Edizione digitale}

URL: http://journals.openedition.org/studifrancesi/6447

DOI: 10.4000/studifrancesi.6447

ISSN: 2421-5856

\section{Editore}

Rosenberg \& Sellier

\section{Edizione cartacea}

Data di pubblicazione: 1 novembre 2010

Paginazione: 594-595

ISSN: 0039-2944

\section{Notizia bibliografica digitale}

Carminella Biondi, «Lilian Thuram, Mes étoiles noires. De Lucy à Barack Obama», Studi Francesi [Online], 162 (LIV | III) | 2010, online dal 30 novembre 2015, consultato il 10 janvier 2021. URL: http:// journals.openedition.org/studifrancesi/6447 ; DOI: https://doi.org/10.4000/studifrancesi.6447

Questo documento è stato generato automaticamente il 10 janvier 2021.

\section{(c) (i) (9)}

Studi Francesi è distribuita con Licenza Creative Commons Attribuzione - Non commerciale - Non opere derivate 4.0 Internazionale. 


\title{
Lilian Thuram, Mes étoiles noires. De Lucy à Barack Obama
}

\author{
Carminella Biondi
}

\section{NOTIZIA}

LILIAN THURAM, Mes étoiles noires. De Lucy à Barack Obama, Paris, Phillipe Rey, 2010, pp. 400.

1 Agli inizi dell'Ottocento, l'abbé Grégoire, abolizionista e strenuo difensore dell'uguaglianza razziale, aveva pubblicato un corposo studio, intitolato De la littérature des nègres (1808), in cui presentava alcuni personaggi neri che si erano distinti nel mondo delle arti e delle scienze. Un lavoro straordinario, che però conteneva un pericoloso tallone d'Achille: i personaggi di cui l'Abate si occupa sono infatti tutti neri sradicati dalla loro terra e portati schiavi nelle colonie americane, che hanno dimostrato la loro abilità nell'elaborazione di opere letterarie o scientifiche che rispondevano al canone della cultura occidentale. Un terreno su cui, pur con tutti i loro meriti, non potevano che essere svantaggiati. Per i critici non fu pertanto difficile cogliere i punti deboli delle argomentazioni utilizzate da Grégoire e la fragilità degli esempi da lui citati (vedi in particolare Cri des colons di de Tussac, pubblicato lo stesso anno). L'autore del saggio qui presentato è invece ben consapevole di questi limiti, quando inserisce fra le "le sue stelle nere", la poetessa del Settecento Phillis Wheatley (che faceva parte anche dell'Olimpo nero dell'abbé Gregoire): «Phillis Wheatley [...] devient "légalement" la première poétesse noire de l'histoire de la littérature... pour les Occidentaux! Je le précise, parce que l'histoire des Noirs commence, pour une grande majorité de nos contemporains, à l'esclavage. Nul doute qu'il y eut des milliers de poétesses noires avant Phillis Wheatley...» (p. 94).

2 Lilian Thuram, nato in Guadalupa, giocatore di pallone noto a livello internazionale, ha sentito pesare su di sé, e in generale sugli uomini di colore, una esplicita o subdola discriminazione razziale e ha capito che nessuna legge può cambiare le cose, se non si cambiano gli immaginari: «Si nous voulons vriement changer notre société, lutter 
contre le racisme, ce n'est pas sur la discrimination positive ni sur le communautarisme qu'il faut compter. Seul le changement de nos imaginaires peut nous rapprocher et faire tomber nos barrières culturelles; là seulement nous pourront dépasser l'obstacle majeur qui se cache derrière des mots comme "minorité visible", "diversité" - les "vous" et "nous" déterminés par la couleur de la peau» (p.9). Per arrivare a questo traguardo bisogna sforzarsi di riscrivere, in maniera più obiettiva, la storia del mondo. L'autore spera di contribuirvi con questo libro (per la stesura del quale si è avvalso della collaborazione di Bernard Fillaire), che, come indica il sottotitolo, parte dalla mitica antenata Lucy «notre grand-mère africaine», per giungere alla realtà-mito di oggi rappresentata da Barack Obama (il cui avvento è già stato salutato dagli scrittori martinicani Édourad Glissant e Patrick Chamoiseau, in L'intraitable beauté du monde. Adresse à Barack Obama, 2008).

Mes étoiles noires è composto di quarantacinque medaglioni che presentano altrettanti personaggi (neri o mulatti) noti, meno noti e sconosciuti, che si sono distinti nei campi più diversi, in terra d'Africa e nelle terre della diaspora. Non credo sia tanto importante soffermarsi su autori noti, come Césaire e Fanon, il mulatto Pus`kin o su personaggi altrettanto famosi come Lumumba, Martin Luther King o Malcom X, ecc. che sono stati oggetto di studi ben più approfonditi, ma che non potevano essere per questo esclusi dall'Olimpo. Più interessante è la presenza di personaggi assai meno conosciuti, neri o mulatti, come il musicista Saint-Georges o il pittore Lethière, e soprattutto quella di alcune donne assolutamente sconosciute anche se i libri di storia, le relazioni di viaggio e persino qualche vecchio romanzo hanno riservato loro un certo spazio. Alludo ad Anne Zingha, che è stata regina d'Angola nel primo Seicento, a Dona Beatrice, «la combattante du renouveau» (p. 45), vissuta in Congo a cavallo fra Sei e Settecento, a la "mulâtresse" Solitude, che ha combattuto, nel 1802, a fianco di Louis Delgrès, in Guadalupa, ed è morta come lui per la difesa della libertà dei neri. La sua epopea è già stata raccontata nel bel romanzo di André Schwarz-Bart (autore noto per aver vinto il Goncourt nel 1959 con L'ultimo dei giusti), La mulâtresse Solitude (1972), ma forse non tutti sanno che si tratta di un personaggio realmente esistito. Tanti altri ritratti, pur meritevoli di attenzione, saranno trascurati in questa rapida presentazione, ma non si può certo ignorare, l'ultimo della serie, quello che sembra rappresentare il punto di arrivo di un lungo percorso che parte dalla preistoria, il ritratto di Barack Obama. Un ritratto, forse inevitabilmente, un po' di maniera, ma dal quale emerge con evidenza di quali speranze si carichi, e non solo per il mondo nero, l'elezione di Obama alla presidenza degli Stati-Uniti: «Je me dis que cette élection va changer bien des choses dans l'imaginaire des peuples et représenter un formidabile encouragement à l'éducation contre le racisme» (p.365). Thuram spera che la stella di Obama contribuisca in maniera forte a superare quei pregiudizi che hanno, nei fatti, impedito l'estensione al mondo nero sia della Dichiarazione dei diritti dell'uomo e del cittadino del 1789, che della Dichiarazione universale dei diritti dell'uomo del 1948. Il volume si conclude con alcune pagine dello psichiatra per bambini Gilles-Marie Valet («Des mots qui libèrent l'avenir») sui pericoli insiti nella scelta di non raccontare a un bambino le proprie origini, come in quella di privare un popolo delle proprie radici. Un'introduzione agile e godibile, ma non scontata, all'altra faccia della Storia. 